\title{
Recent Applications of $p$-adic Methods to Commutative Algebra
}

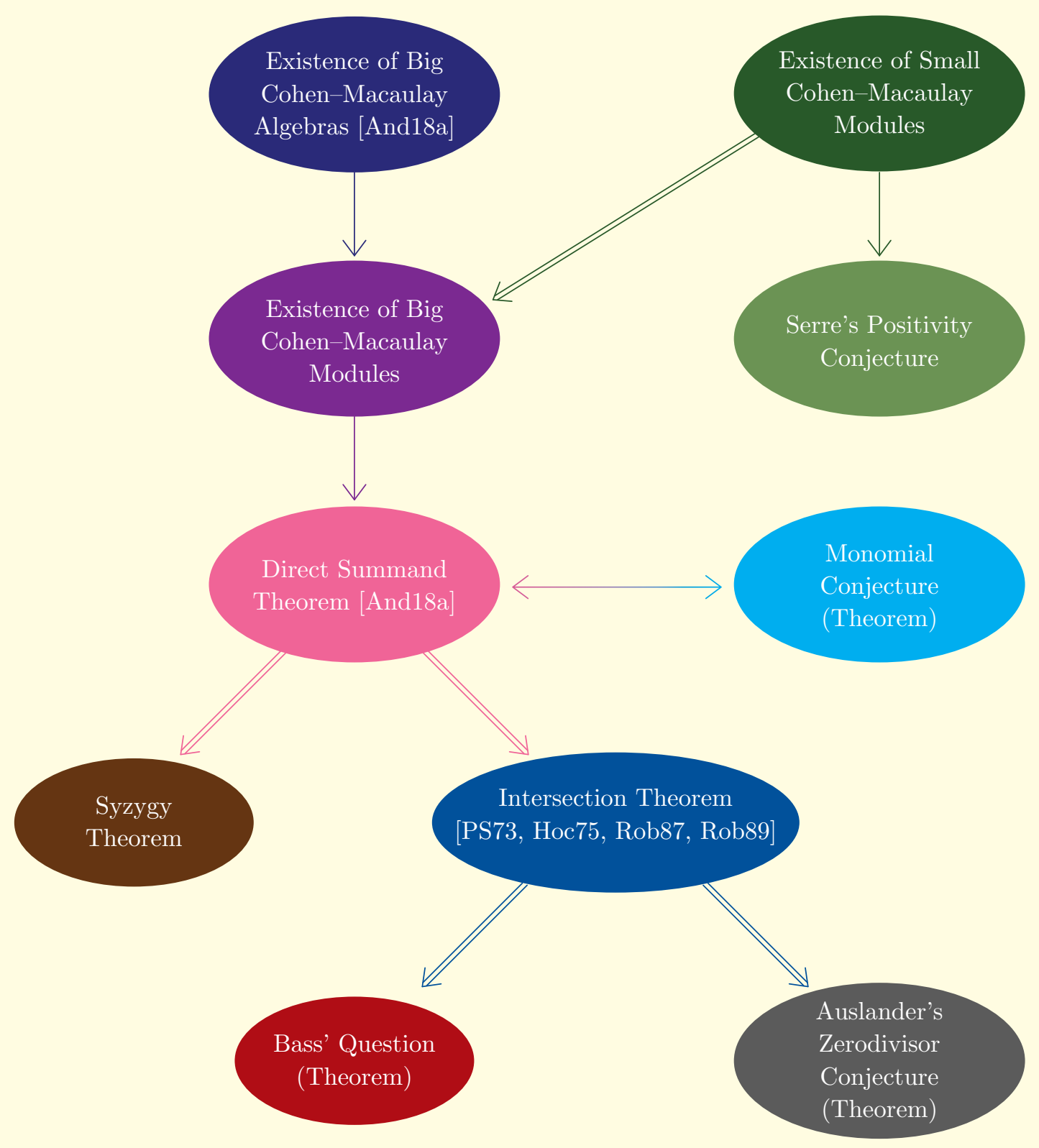

\section{Linquan Ma and Karl Schwede}


Suppose that $k$ is a field and $R=k\left[x_{1}, \ldots, x_{n}\right] / I$ is a commutative polynomial ring over $k$ modulo an ideal $I$. In other words, it is a finitely generated commutative $k$ algebra. Additionally assume that $R$ is also an integral domain. Emmy Noether's celebrated Normalization Theorem proves that inside $R$, there always exists a polynomial subring

$$
A=k\left[t_{1}, \ldots, t_{d}\right] \subseteq R
$$

where the $t_{i}$ are algebraically independent (have no relations between them) satisfying the following property: the extension of rings $A \subseteq R$ makes $R$ into a finitely generated module over the polynomial ring $A=k\left[t_{1}, \ldots, t_{d}\right]$. For example, in $R=k[x, y] /\left(y^{2}-x^{3}\right)$ we have the subring $A=k[x]$ (or $B=k[y])$.

Consider the induced finite extension of fraction fields

$$
k\left(t_{1}, \ldots, t_{d}\right)=K(A) \subseteq K(R) .
$$

By viewing $K(R)$ as a vector space over $K(A)$, each element $u$ of $K(R)$ acts via multiplication

$$
\times u: K(R) \longrightarrow K(R)
$$

and so we can take its trace, which is then an element of $K(A)$. This induces a map

$$
\operatorname{Tr}: K(R) \longrightarrow K(A) .
$$

It is not difficult to verify $\operatorname{Tr}(R) \subseteq A$, and so one obtains a map

$$
\operatorname{Tr}: R \rightarrow A=k\left[t_{1}, \ldots, t_{d}\right] .
$$

Since the trace is a sum of the diagonal matrix entries, the composition

$$
A \subseteq R \stackrel{\operatorname{Tr}}{\rightarrow} A
$$

is multiplication by the extension degree $n:=[K(R)$ : $K(A)$ ]. If $k$ has characteristic zero (or more generally if the characteristic does not divide the extension degree $n=$ $[K(R): K(A)])$, then the composition

$$
A \subseteq R \stackrel{\frac{1}{n} \cdot \operatorname{Tr}}{\longrightarrow} A
$$

Linquan Ma is an assistant professor at Purdue University. His email address is ma326@purdue.edu.

Karl Schwede is a professor at the University of Utah. His email address is schwede@math.utah.edu.

The first named author was supported in part by NSF Grant \#1836867/ 1600198.

The second named author was supported in part by NSF CAREER Grant DMS \#1252860/1501102 and NSF grant \#1801849.

This material is based upon work supported by the National Science Foundation under grant No. DMS-1440140 while the authors were in residence at the Mathematical Sciences Research Institute in Berkeley, California, during the Spring 2019 semester.

Communicated by Notices Associate Editor Daniel Krashen.

For permission to reprint this article, please contact:

reprint-permission@ams.org.

DOI: https://doi.org/10.1090/noti1896 is the identity. Hence, if $M=\operatorname{ker}\left(\frac{1}{n} \cdot \operatorname{Tr}\right)$, then $R \cong$ $A \oplus M$ as an $A$-module. In this case, we say that $R$ has an $A$-summand and that $A \subseteq R$ splits.

Consider now a more general setup. Suppose that

$$
A \subseteq R
$$

is a finite extension of Noetherian integral domains. We ask when $R$ has an $A$-summand, i.e., when is $R \cong A \oplus$ $M$ for some $M$ ? In [Hoc73] Hochster conjectured that if $A$ is a Noetherian regular ring ${ }^{1}$ and $R \supseteq A$ is any extension ring that is finite as an $A$-module (henceforth, a finite extension), then $R$ has an $A$-summand.

This was the direct sum-

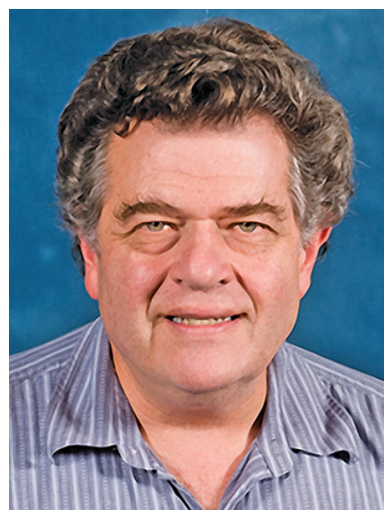

Melvin Hochster. mand conjecture, now André's theorem [And18a], and it was one of the central and guiding questions of commutative algebra over the past half-century.

Theorem 1 (Direct Summand Theorem). Suppose $A$ is a Noetherian regular ring and $R \supseteq A$ is a finite extension, then $A \hookrightarrow$ $R$ splits as a map of $A$-modules. In other words, $R$ has an $A$ summand.

We observed above that the theorem holds if $A$ is a polynomial ring over a field of characteristic zero. In fact, the same argument works if $A$ is any regular domain (or even normal ${ }^{2}$ domain) containing the rational numbers $\mathbb{Q}$. Hochster proved in [Hoc73] that Theorem 1 also holds if $A$ is a regular ring containing the finite field $\mathbb{F}_{p}=\mathbb{Z} / p \mathbb{Z}$ (e.g., $A=\mathbb{F}_{p}\left[x_{1}, \ldots, x_{d}\right]$ ). The methods that go into this and the areas of research they spawned are the topic of "The Direct Summand Conjecture and Singularities in Characteristic $p . "$

Example 2. The finite extension $A=\mathbb{Q}\left[t^{2}, t^{3}\right] \hookrightarrow R=$ $\mathbb{Q}[t]$ does not split. If there was a splitting $\phi: R \longrightarrow A$, it must send $1 \mapsto 1$ and therefore it must also send $t^{2}$ and $t^{3}$ to themselves. But $t^{3}=\phi\left(t^{3}\right)=\phi\left(t^{2} \cdot t\right)=t^{2} \phi(t)$ and so $\phi(t)=t$, which does not exist in $A$. Note $A$ is not normal.

In a recent breakthrough [And18b, And18a], André solved the conjecture in the mixed characteristic ${ }^{3}$ case, using Scholze's theory of perfectoid algebras and spaces [Sch12]. This will be the topic of the section "Perfectoid Algebras and Ingredients in the Mixed Characteristic Proof."

\footnotetext{
$1_{\text {Regular rings are natural generalizations of polynomial rings over fields and also include }}$ rings such as $\mathbb{Z}\left[x_{1}, \ldots, x_{d}\right]$.

${ }^{2}$ Meaning $A$ is integrally closed in its fraction field $K(A)$. In particular, if $f \in K(A)$ satisfies a monic polynomial with coefficients in $A$, then $f \in A$.

${ }^{3} A$ ring $A$ has mixed characteristic if it contains the integers $\mathbb{Z}$ as a subring, and there is some prime $p \in \mathbb{Z} \subseteq A$ that is not invertible in $A$.
} 


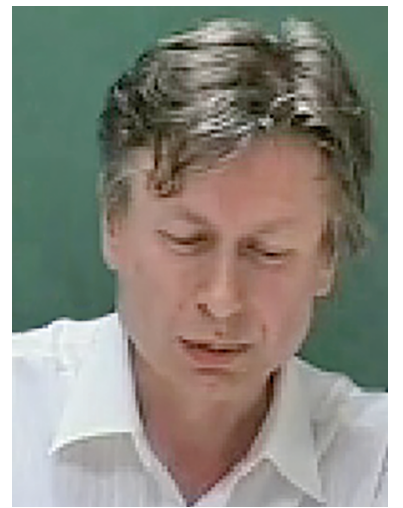

Yves André.
Previously, the best case that was known was the case when $A$ is a regular ring of dimension $\leq 3$, which is due to Heitmann [Hei02]. In the mixed characteristic setting, Bhargav Bhatt and Ofer Gabber also made substantial contributions to these circles of ideas [Bha14a, Bha18, Gab18].

The methods of André's proof have also been used to prove generalizations of the direct summand theorem, notably the existence of big Cohen-Macaulay algebras and the derived direct summand theorem, see [And18a, And18c, Bha18, Gab18, HM18, Shi17]. We expect that the existence of big Cohen-Macaulay algebras will stimulate further study of $f$ in mixed characteristic: In fact, they can be thought of as a tool that replaces certain aspects of Hironaka's resolution of singularities from characteristic zero algebraic geometry, as explained in [MS18b]. We will discuss big Cohen-Macaulay algebras and singularities in mixed characteristic in the section "Big Cohen-Macaulay Algebras and Singularities in Mixed Characteristic." As an application of these ideas, in our final section, "An Application to Symbolic Powers," we discuss a result on uniform growth of symbolic powers of ideals [MS18a].

Homological Conjectures. The Homological Conjectures in commutative algebra are a network of conjectures relating

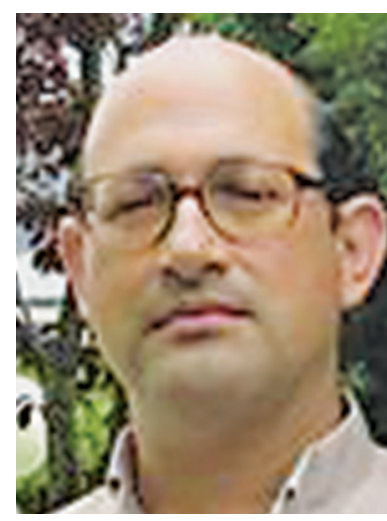
various homological properties of a commutative ring with its internal ring structure. They have generated a tremendous amount of activity over the last fifty years. The following is a diagram of homological conjectures, which is part of Hochster's 2004 diagram [Hoc04] (one sees that the Direct Summand Conjecture/Theorem lies in the heart). Most of these conjectures are now completely resolved thanks to the work of André and oth-

ers.

Some of these implications are highly nontrivial: For example, the fact that the Direct Summand Theorem implies the Syzygy Theorem and the Intersection Theorem was due to Hochster [Hoc83], ${ }^{4}$ and that the Intersection Theorem implies Bass' Question and Auslander's Conjec-

${ }^{4}$ See also [Dut87] for other interesting connections between the homological conjectures.

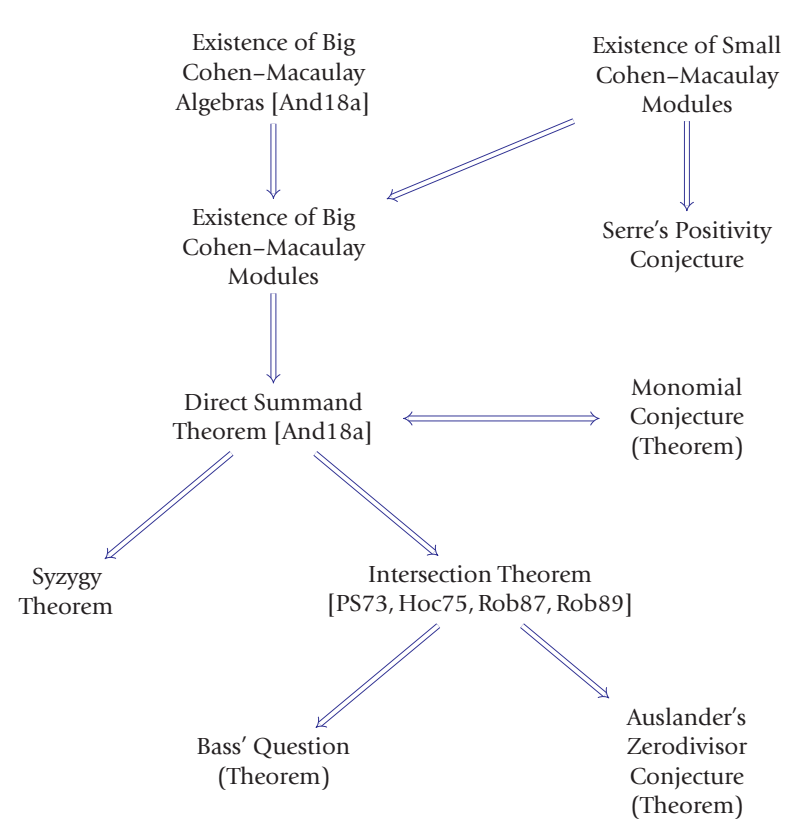

ture was proved by Peskine-Szpiro [PS73]. We note that many of the early homological conjectures are solved in mixed characteristic, thanks to Roberts' proof of the Intersection Theorem using localized Chern characters [Rob87, Rob89]. We also mention that there are various stronger forms of some of these conjectures that are proved based on André's work, see for example [And18c, AIN18, Gab18, HM18].

We want to highlight that, despite the recent breakthroughs in mixed characteristic, Serre's Positivity Conjecture on intersection multiplicity is still wide open in the ramified mixed characteristic case. To this date, the

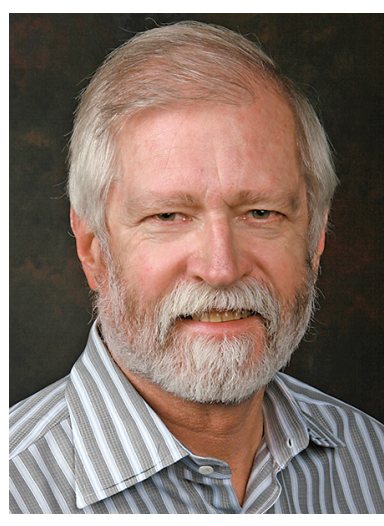

Paul C. Roberts. most important progress towards Serre's Conjecture is due to Gabber, see [Hoc97]. We refer the reader to [Hoc17] for a recent extensive survey on Serre's Conjecture and other (old and new) homological conjectures and theorems.

We end the introduction by briefly discussing one of the homological theorems in the diagram above.

Theorem 3 (The Syzygy Theorem). Let $(R, \mathfrak{m})$ be a CohenMacaulay (or even regular) local domain and let $M$ be a nonfree finitely generated $R$-module. If $M$ is a $k$-th syzygy module of finite projective dimension, ${ }^{5}$ then the rank of $M$ is at least $k$.

For instance, the first syzygy module is a submodule of

\footnotetext{
${ }^{5}$ This means $M \cong \operatorname{Image}\left(\delta_{k}\right)$ in a finite free resolution $0 \rightarrow F_{n} \rightarrow \cdots \rightarrow F_{k} \stackrel{\delta_{k}}{\longrightarrow}$ $\cdots \longrightarrow F_{0} \longrightarrow N \longrightarrow 0$ of a finitely generated $R$-module $N$.
} 
a free module $F_{0}$, so its rank is at least one (i.e., it cannot be torsion). Theorem 3 is a huge generalization to higher syzygy modules.

Theorem 3 was first proved by Evans-Griffith when $R$ contains a field [EG81] based on earlier work of Hochster [Hoc75]. The fact that it follows from Theorem 1 in mixed characteristic is a result of Hochster [Hoc83]. So this is now a theorem by André's work.

Theorem 3 itself has many unexpected consequences. For instance, it has connections to Horrock and Hartshorne's question on the cohomology of vector bundles of small rank on $\mathbb{P}^{n}$ [Har79].

The Direct Summand Conjecture and Singularities in Characteristic $p$

Suppose now that $A$ is a regular Noetherian domain and $R \supseteq A$ is a finite extension that is also a domain. The fraction field extension

$$
K(A) \subseteq K(R)
$$

is separable if and only if the map $\operatorname{Tr}: R \longrightarrow A$ is nonzero. So to solve the direct summand conjecture, we cannot expect to use the field trace as we did when $A$ contains $\mathbb{Q}$.

For an arbitrary ring $A$ that contains $\mathbb{F}_{p}$, we have the Frobenius map (which is a ring homomorphism):

$$
F: A \longrightarrow A \quad a \longmapsto a^{p} .
$$

We can iterate the Frobenius map: we label the $e$-fold selfcomposition $F^{e}: A \longrightarrow A$ and observe it sends $a \mapsto a^{p^{e}}$. One of the first issues one runs into when working with the Frobenius is that it can be difficult to distinguish the source and target of the map as they are the same ring. We explain one way to handle this issue. In the case that $A$ is an integral domain, we let $A^{1 / p^{e}}$ denote the ring of $p^{e}$ th roots of elements of $A$ embedded inside the algebraic closure of the fraction field, in other words:

$$
A^{1 / p^{e}}:=\left\{x \in \overline{K(A)} \mid x^{p^{e}} \in A\right\} .
$$

The ring $A^{1 / p^{e}}$ is abstractly isomorphic to $A$ via the map $A^{1 / p^{e}} \longrightarrow A$ which sends $b \mapsto b^{p^{e}}$. This isomorphism identifies the inclusion

$$
A \subseteq A^{1 / p^{e}}
$$

with Frobenius, and it provides us with a convenient way of distinguishing the source and target of the Frobenius map.

The proof of the direct summand conjecture in characteristic $p>0$ we present here follows from [Hoc73] in spirit. We begin by proving the following lemma (we use formal power series instead of the polynomial ring, but the idea is the same); this lemma also motivates further investigations on characteristic $p>0$ singularities.

Lemma 4. Suppose $k$ is a perfect field of positive characteristic and $A=k \llbracket x_{1}, \ldots, x_{d} \rrbracket$ is the formal power series ring. Then for every nonzero $c \in A$, there exists an $e>0$ such that the A-module map $A \stackrel{1 \mapsto c^{1 / p^{e}}}{\longrightarrow} A^{1 / p^{e}}$ splits.

Proof. For any nonzero $c \in A$, by looking at terms of $c$ of minimal degree, there exists an $e$ so that

$$
c \notin\left(x_{1}^{p^{e}}, \ldots, x_{d}^{p^{e}}\right) .
$$

In other words, $c^{1 / p^{e}} \notin\left(x_{1}, \ldots, x_{d}\right) \cdot A^{1 / p^{e}}$. Thus the image of $c^{1 / p^{e}}$ is nonzero in the $k$-vector space

$$
\frac{A^{1 / p^{e}}}{\left(x_{1}, \ldots, x_{d}\right) \cdot A^{1 / p^{e}}} .
$$

By Nakayama's lemma, $C$ can be chosen as part of a basis for the free $A$-module $A^{1 / p^{e}}$. Hence there is a map $\psi$ : $A^{1 / p^{e}} \rightarrow A$ such that $\psi\left(c^{1 / p^{e}}\right)=1$, which proves the lemma.

Rings that satisfy the conclusion of Lemma 4 are called strongly F-regular (see Definition 7). We will discuss them more in what follows.

Theorem 5. If $A$ is a Noetherian regular ring of characteristic $p>0$, then any finite ring extension $A \subseteq R$ splits.

Proof. By standard commutative algebra techniques, we may assume that $A \cong k \llbracket x_{1}, \ldots, x_{d} \rrbracket$ where $k$ is perfect and that $R$ is an integral domain.

For any integer $e>0$ and any $A$-linear map $\phi: A^{1 / p^{e}}$ $\longrightarrow A$ we consider the commutative diagram:

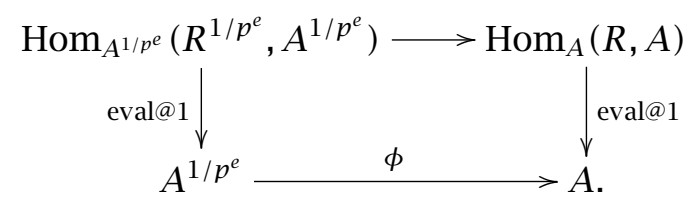

The top horizontal map is obtained by restricting to $R$ the domain of an element $\psi \in \operatorname{Hom}_{A^{1 / p^{e}}}\left(R^{1 / p^{e}}, A^{1 / p^{e}}\right)$ and then post-composing with $\phi$. Since the vertical maps are evaluation at 1 , to show that $A \longrightarrow R$ splits it is enough to show that the right vertical map surjects (then there exists $\psi \in \operatorname{Hom}_{A}(R, A)$ so that $\left.\psi(1)=1\right)$. Because $\operatorname{Hom}_{A}(R, A) \stackrel{\text { eval@1 }}{\longrightarrow} A$ surjects generically, i.e., it is surjective if we tensor with the fraction field of $A$, we can choose a nonzero $c$ in the image of $\operatorname{Hom}_{A}(R, A) \stackrel{\text { eval@1 }}{\longrightarrow} A$. It follows that $c^{1 / p^{e}}$ is in the image of the left vertical map (for any $e>0$ ). Next, using Lemma 4 , we choose $e>0$ and an $A$-linear map $\phi: A^{1 / p^{e}} \rightarrow A$ sending $c^{1 / p^{e}} \rightarrow 1$. The commutative diagram implies that the composition from the upper left to the lower right surjects, and hence so does $\operatorname{Hom}_{A}(R, A) \stackrel{\text { eval@1 }}{\longrightarrow} A$.

\footnotetext{
${ }^{6}$ One common choice of basis is that made up of all elements $x_{1}^{j_{1} / p^{e}} \cdots x_{d}^{j_{d} / p^{e}}$ where each $j_{i}$ varies between 0 and $p^{e}-1$.
} 
Philosophy. We take a step back to think through this proof. In characteristic $p>0$, the trace map does not always lead to splittings, even for separable extensions due to the presence of what is called wild ramification. Roughly speaking, we get around this by choosing an element $c \in$ $A$ over which $A \subseteq R$ is ramified. By taking $1 / p^{e}$ th roots of $c$ (or in other words using Frobenius), we minimize the ramification until it almost goes away and so that extension $A \subseteq R$ splits.

We also give a new example of a ring with a non-split extension.

Example 6. Consider $k=\overline{\mathbb{F}_{p}}$, the algebraic closure of the finite field $\mathbb{F}_{p}=\mathbb{Z} / p \mathbb{Z}$. We form the ring $A=k[x, y, z] /$ $\left(z y^{2}-x(x-z)(x+\lambda z)\right)$, defining the affine cone over a (projective) elliptic curve $E$ (for example, one could take $\lambda=1$ ). If the characteristic $p>0$ is such that the curve is supersingular, then the (absolute) Frobenius map $F$ : $A \longrightarrow A$ (which sends $r \mapsto r^{p}$ for all $r \in R$ ) does not split. If $p>0$ is such that the curve is ordinary, then there exists a degree $p$ étale map $E^{\prime} \longrightarrow E$ between elliptic curves [Sil09, Chapter V, Theorem 3.1]. This gives a finite extension $A \hookrightarrow A^{\prime}$ of the rings corresponding to the cones. This extension is not étale at the cone point(s) and in fact is not split. In either case $A$ has a finite extension $A \hookrightarrow R$ that is not split.

Singularities in characteristic $p>0$. The techniques we used to prove the direct summand conjecture in characteristic $p>0$ have led to a vigorous study of singularities in characteristic $p>0$ (typically under the names tight closure theory and Frobenius splitting theory).

Definition 7 (Strongly $F$-regular singularities). A Noetherian domain $S$ containing $\mathbb{F}_{p}$ such that $S^{1 / p}$ is a finitely generated $S$-module is called strongly $F$-regular if for any nonzero $c \in S$, there is an $e>0$ and $\phi: S^{1 / p^{e}} \longrightarrow S$ such that $\phi\left(c^{1 / p^{e}}\right)=1$. In this case, the singularities of Spec $S$ are called strongly F-regular singularities.

The proof we gave in Theorem 5 shows that strongly $F$ regular rings are direct summands of all their finite extensions. In fact, a Noetherian domain that is a direct summand of every finite extension is called a splinter. Thus we have:

$$
\text { (Strongly } F \text {-regular ring) } \Rightarrow \text { (Splinter) }
$$

The converse is open except in the case that the ring is (close to) Gorenstein [HH94, Sin99]. In fact, the converse would imply arguably the most studied question in characteristic $p>0$ commutative algebra: that a weakly $F$ regular ring ${ }^{7}$ is strongly $F$-regular.

\footnotetext{
$\overline{7_{A} \text { ring is called weakly } F \text {-regular if all of its ideals are tightly closed [HH90]; we will not }}$ delve into these definitions here, however.
}

Example 8. In Example 6, we saw that the affine cone over an elliptic curve is not a splinter. The ring $\overline{\mathbb{F}_{p}}[x, y, z] /$ $\left(x y-z^{2}\right)$ is strongly $F$-regular, and hence it is a splinter. The fact that it is strongly $F$-regular most easily follows from the fact that it is a direct summand of a regular ring.

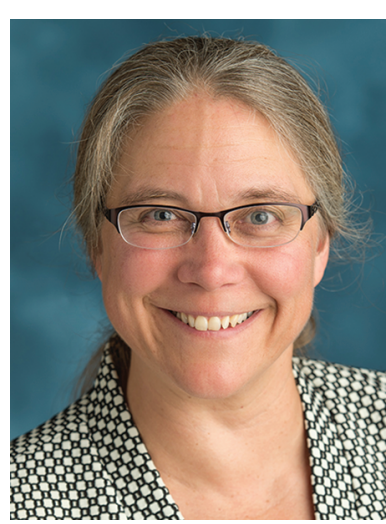

Karen E. Smith.
Strongly $F$-regular singularities are intimately tied to singularities that appear in complex algebraic geometry. Roughly speaking, a complex algebraic variety $X$ has rational singularities if its line bundles have the same sheaf cohomology as the pullbacks of those line bundles to a resolution of singularities. ${ }^{8}$

A refinement of rational singularities is log terminal singularities (rational singularities whose finite étale in codimension 1 covers also have rational singularities). Rational singularities are exactly the same as log terminal singularities on hypersurfaces (and more generally, on Gorenstein varieties).

What is really surprising, given their completely disjoint definitions, is that log terminal singularities are essentially the same as strongly $F$-regular singularities, modulo reduction to characteristic $p>0$. Specifically, suppose that a chart $U$ on $X$ is given as the spectrum of $R_{\mathbb{C}}=\mathbb{C}\left[x_{1}, \ldots, x_{d}\right]$ $/ I_{\mathbb{C}}$. Suppose for simplicity that all the coefficients of the generators of the ideal $I_{\mathbb{C}}$ live in $\mathbb{Z}$. We can reduce $R_{\mathbb{C}}$ to characteristic $p>0$ by taking the coefficients of the generators of the ideal $I_{\mathbb{C}}$ modulo some $p>0$. For example:

$$
f=x^{2}+101 x y-7 \sim \bmod 5 \leadsto x^{2}+x y+3 .
$$

For each $p>0$, this gives us a ring $R_{p}=\mathbb{F}_{p}\left[x_{1}, \ldots, x_{d}\right] / I_{p}$.

Theorem 9 ([HW02,Har98,Smi97,MS97]). $U=\operatorname{Spec} R_{\mathbb{C}}$ has log terminal singularities if and only if for all $p \gg 0, R_{p}$ has strongly F-regular singularities.

In fact, this connection is a small part of a large dictionary where notions from higher dimensional complex algebraic geometry correspond to concepts involving the Frobenius map.

Definition 10 (Derived splinters). A Noetherian domain $S$ is called a derived splinter if for every proper surjective map $\pi: Y \longrightarrow X=\operatorname{Spec} S$, the induced map $\mathcal{O}_{X} \longrightarrow$ $R \pi_{*} \mathcal{O}_{Y}$ splits in the derived category of $\mathcal{O}_{X}$-modules.

\footnotetext{
${ }^{8}$ A resolution of singularities is a proper map $\pi: Y \rightarrow X$ of varieties such that $Y$ is nonsingular and $\pi$ is birational, which means it is an isomorphism "almost everywhere" (i.e., on a Zariski open and dense subset).

${ }^{9}$ essentially covering spaces
} 


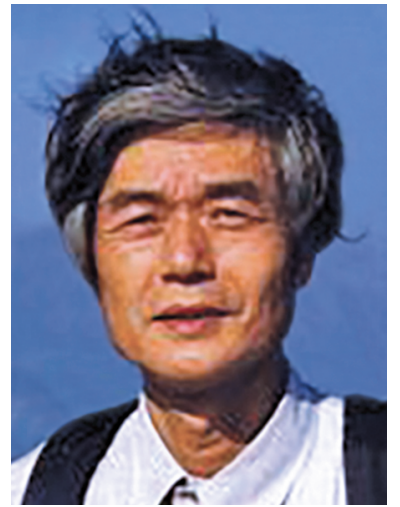

Kei-ichi Watanabe.
It is a theorem of Bhatt that for rings of finite type over fields of characteristic zero, rational singularities are exactly derived splinters [Bha12] (and also see [Kov00]). But, in [Bha12], Bhatt also shows that derived splinters in characteristic $p>0$ are exactly the same as splinters. Both these results strongly suggest that regular rings should be derived splinters in general. This derived statement is an extension of the direct summand conjecture (since any finite extension of ring $A \subseteq R$ induces a proper surjective map $\operatorname{Spec} R \longrightarrow \operatorname{Spec} A$ ). In [Bha18], applying some of Andrés ideas, Bhatt gives a simplified proof of Theorem 1 and also proves this derived version.

Theorem 11 (The Derived Direct Summand Theorem). Any Noetherian regular ring is a derived splinter.

\section{Perfectoid Algebras and Ingredients in the Mixed Characteristic Proof}

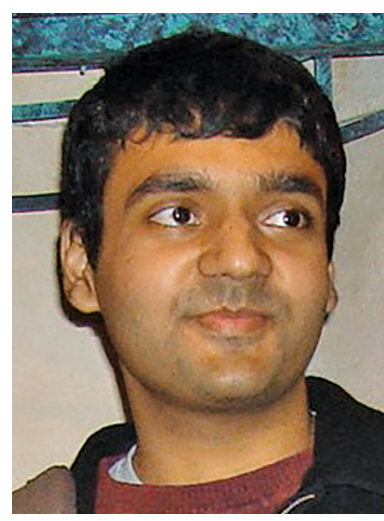

Now we move to the discussion of the proof of the Direct Summand Theorem (Theorem 1) in mixed characteristic, which uses perfectoid techniques. Consider a Noetherian local ring $A$ with maximal ideal m. As before, we say that $A$ has mixed characteristic $(0, p)$ if $A$ has characteristic 0 and $A / \mathfrak{m}$ has prime characteristic $p>0$. For example, the ring of $p$-adic integers $\mathbb{Z}_{p}$ (the ring of formal power series in $p$ ) has maximal ideal generated by $p$, its residue Bhargav Bhatt. field is $\mathbb{F}_{p}$, while its fraction field $\mathbb{Q}_{p}$ has characteristic 0 .

We use $\mathbb{Q}_{p}$ to construct our first example of a perfectoid ring. Begin by adjoining all $p^{e}$ th roots of $p$ to $\mathbb{Q}_{p}$ to form $\mathbb{Q}_{p}\left(p^{1 / p^{\infty}}\right)$. We $p$-adically complete and call the resulting ring $K=\mathbb{Q}_{p} \widehat{\left(p^{1 / p^{\infty}}\right)}$. This field $K$ is a typical example of a perfectoid field. The field $K$ contains a subring $K^{\circ}=\mathbb{Z}_{p} \widehat{\left[p^{1 / p^{\infty}}\right]}$, which is its "ring of integers." $K^{\circ}$ is a typical example of an integral perfectoid ring. For the rest of this section, we fix $K$ and $K^{\circ}$ as above. We now give a definition of a perfectoid algebra.

Definition 12 (Perfectoid algebras [Sch12, BMS18, And18c]). A perfectoid $K$-algebra is a Banach $K$-algebra
$R$ such that the set of power-bounded elements ${ }^{10} R^{\circ} \subseteq R$ is bounded and the Frobenius is surjective on $R^{\circ} / p$. A $K^{\circ}$-algebra $S$ is called integral perfectoid if it is $p$-adically complete, $p$-torsion free, and the Frobenius induces an isomorphism $S / p^{1 / p} \longrightarrow S / p$.

If $R$ is a perfectoid $K$-algebra, then the ring of powerbounded elements $R^{\circ}$ is integral perfectoid, and if $S$ is integral perfectoid, then $S[1 / p]$ is a perfectoid $K$-algebra, see [Sch12, Theorem 5.2].

We give examples of integral perfectoid algebras. It is important to note that these algebras are never Noetherian. As before, $\hat{\bullet}$ denotes the $\boldsymbol{p}$-adic completion of $\bullet$.

Example 13. (a) $\left.K^{\circ}=\mathbb{Z}_{p} \widehat{\left[p^{1 / p^{\infty}}\right.}\right]$.

(b)

$$
\begin{aligned}
K^{\circ}\left\langle x_{2}^{1 / p^{\infty}}, \ldots, x_{d}^{1 / p^{\infty}}\right\rangle \\
\quad:=\mathbb{Z}_{p}\left[p^{1 / p^{\infty}}\right]\left[x_{2}^{1 / p^{\infty}}, \ldots, x_{d}^{1 / p^{\infty}}\right] .
\end{aligned}
$$

(c) $\widehat{R^{+}}$, where $(R, \mathfrak{m})$ is a Noetherian complete local domain of mixed characteristic $(0, p)$ and $R^{+}$is the integral closure of $R$ in an algebraic closure of its fraction field.

Although we will not use it in this survey, a key idea in the theory of perfectoid algebras (and spaces) is that numerous questions can be studied via tilting. This can turn a mixed characteristic question (or ring) into one in positive characteristic, and vice versa. This principle is used extensively (behind the scenes) in what follows. For more discussion, see for instance [Bha14b, Sch12].

A key part of the general theory of perfectoid rings is that we can talk about "almost mathematics" (appearing originally in the work of Faltings, [Fal88], also see the work of Gabber and Ramero [GR03]). Roughly speaking, we treat modules that are annihilated by the ideal $\left(p, p^{1 / p}\right.$, $\left.p^{1 / p^{2}}, \ldots\right)=:\left(p^{1 / p^{\infty}}\right) \subseteq K^{\circ}$ as if they were zero (since $p^{1 / p^{e}}$ is almost 1 for $e \gg 0$ ).

Definition 14. Let $S$ be an integral perfectoid $K^{\circ}$-algebra.

(a) An $S$-module $M$ is almost zero if $\left(p^{1 / p^{\infty}}\right) M=0$.

(b) An $S$-module $M$ is almost flat if

$$
\left(p^{1 / p^{\infty}}\right) \operatorname{Tor}_{1}^{S}(M, N)=0
$$

for all $S$-modules $N$.

(c) A short exact sequence of $S$-modules $0 \longrightarrow M \longrightarrow$ $N \longrightarrow N / M \longrightarrow 0$ represented by a class $\eta \in$ $\operatorname{Ext}_{S}^{1}(N / M, M)$ is almost split if

$$
\left(p^{1 / p^{\infty}}\right) \eta=0 \text { in } \operatorname{Ext}_{S}^{1}(N / M, M) .
$$

\footnotetext{
${ }^{10}$ Elements $x$ such that the norm of $x^{n}$ is bounded independent of $n$.
} 
The most crucial result that is relevant to the proof of Theorem 1 is the following theorem, proved by Scholze [Sch12] and independently by Kedlaya-Liu [KL15]. Special cases were first obtained by Faltings [Fal88, Fal02] and Gabber-Ramero [GR04].

Theorem 15 (The Almost Purity Theorem). Suppose $S$ is integral perfectoid and $S[1 / p] \longrightarrow T$ is a finite étale extension. Then the integral closure $S^{\prime}$ of $S$ in $T$ is an almost finite étale extension of $S$. In particular, $S \longrightarrow S^{\prime}$ is almost split.

Here, almost finite étale roughly means that the obstructions to being finite étale are annihilated by $\left(p^{1 / p^{\infty}}\right)$. For our purposes however, we will only need the weaker fact that the map $S \longrightarrow S^{\prime}$ is almost split.

We sketch the proof of the Direct Summand Theorem (Theorem 1) in mixed characteristic. For simplicity, we set $A=\mathbb{Z}_{p}\left[\overline{x_{2}, \ldots}, x_{d}\right]$. Our goal is to show that every finite extension $A \subseteq R$ splits.

The case $A[1 / p] \longrightarrow R[1 / p]$ is finite étale. We let

$$
A_{\infty}=\mathbb{Z}_{p}\left[p^{1 / p^{\infty}}\right]\left[x_{2}^{1 / p^{\infty}}, \ldots, x_{d}^{1 / p^{\infty}}\right] ;
$$

this is an integral perfectoid algebra. Consider the following diagram:

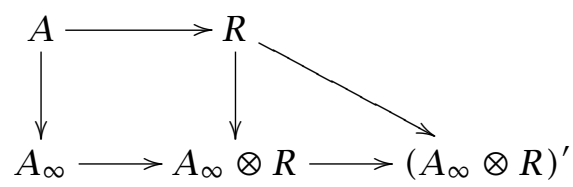

where $\left(A_{\infty} \otimes R\right)^{\prime}$ denotes the normalization of $A_{\infty} \otimes R$ in $A_{\infty}[1 / p] \otimes R$. Since $A_{\infty}[1 / p] \longrightarrow A_{\infty}[1 / p] \otimes R$ is a finite étale extension by base change, Theorem 15 says that the composition

$$
A_{\infty} \longrightarrow A_{\infty} \otimes R \longrightarrow\left(A_{\infty} \otimes R\right)^{\prime}
$$

is almost split and hence $A_{\infty} \longrightarrow A_{\infty} \otimes R$ is almost split. This implies $A \longrightarrow R$ is split, since $A$ is Noetherian and

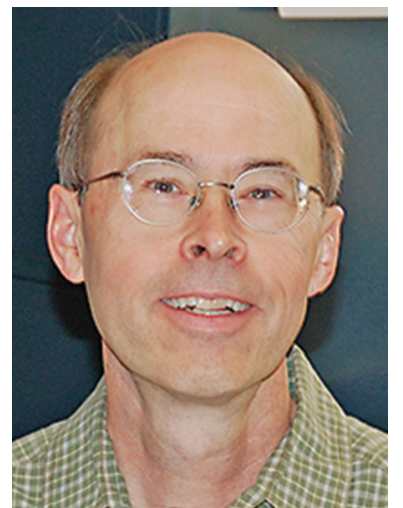

Craig Huneke.
$A \longrightarrow A_{\infty}$ is faithfully flat (we omit the details here, this follows from a standard commutative algebra argument).

The argument above was first observed by Bhatt [Bha14a]. Notice that we only used Theorem 15 for the integral perfectoid algebra $A_{\infty}=\mathbb{Z}_{p}\left[p^{1 / p^{\infty}}\right]\left[x_{2}^{1 / p^{\infty}}, \ldots\right.$, $\left.x_{d}^{1 / p^{\infty}}\right]$. This version is due to Faltings [Fal02].

General case. We now assume that $A[1 / p] \rightarrow R[1 / p]$ is not necessarily étale. But since $A \longrightarrow R$ is "generically étale" (i.e., the extension of the fraction field $K(A) \longrightarrow$
$K(R)$ is finite étale, i.e., separable), we can invert some other element to make $A \longrightarrow R$ étale. Thus we suppose that $A[1 / p g] \longrightarrow R[1 / p g]$ is finite étale for some nonzero element $g$. The main obstruction to "running" the above argument is that Theorem 15 no longer works: $A_{\infty}[1 / p] \otimes R$ is no longer finite étale over $A_{\infty}[1 / p]$. We only know that it becomes finite étale when we further invert $g$. To overcome this difficulty, André proved two remarkable theorems using Scholze's theory of perfectoid spaces:

Theorem 16 ([And18a]). Suppose $S$ is an integral perfectoid algebra and $g \in S$. Then there exists a map $S \longrightarrow \tilde{S}$ of integral perfectoid algebras, such that $g$ admits a compatible system of

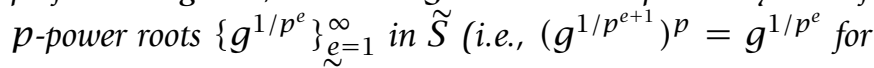
all $e$ ) and that $S \longrightarrow \widetilde{S}$ is almost faithfully flat modulo powers of $p$.

Theorem 17 ([And18b]). Suppose $S$ is integral perfectoid such that $g \in S$ has a compatible system of $p$-power roots in $S$, and $S[1 / p g] \longrightarrow T$ is a finite étale extension. Then the integral closure of $S$ in $T$ is $(p g)^{1 / p^{\infty}}$-almost finite étale over $S$ modulo powers of $p$.

We return to the proof of Theorem 1. As before, we set $A_{\infty}=\mathbb{Z}_{p}\left[p^{1 / p^{\infty}}\right]\left[x_{2}^{1 / p^{\infty}}, \ldots, x_{d}^{1 / p^{\infty}}\right]$. We apply Theorem 16 to construct $A_{\infty} \longrightarrow A_{\infty, \infty}:=\tilde{S}$ such that $g$ has a compatible system of $p$-power roots in $A_{\infty, \infty}$ and such that $A_{\infty, \infty}$ is almost faithfully flat over $A_{\infty} \bmod$ powers of $p$. Consider the following diagram:

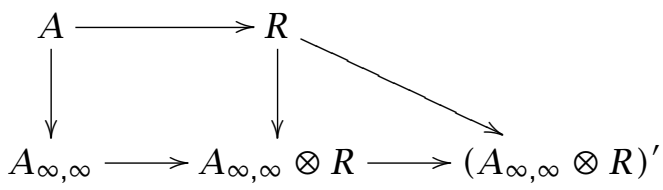

where $\left(A_{\infty, \infty} \otimes R\right)^{\prime}$ denotes the normalization of $A_{\infty, \infty} \otimes$ $R$ in $\left(A_{\infty, \infty} \otimes R\right)[1 / p g]$. Applying Theorem 17 we find that the composition map

$$
A_{\infty, \infty} \longrightarrow A_{\infty, \infty} \otimes R \longrightarrow\left(A_{\infty, \infty} \otimes R\right)^{\prime}
$$

is $(p g)^{1 / p^{\infty}}$-almost split modulo powers of $p$. It follows that $A_{\infty, \infty} \longrightarrow A_{\infty, \infty} \otimes R$ is $(p g)^{1 / p^{\infty}}$-almost split mod powers of $p$. This is enough to conclude that $A \longrightarrow R$ is split by the Noetherianity and $p$-adic completeness of $A$, the faithful flatness of $A_{\infty}$ over $A$, and the almost faithful flatness of $A_{\infty, \infty}$ over $A_{\infty}$ mod powers of $p$ (again some work is required here, but we omit the details).

\section{Big Cohen-Macaulay Algebras and Singularities in Mixed Characteristic}

Let $(R, \mathfrak{m})$ be a Noetherian local ring. Recall that a system of parameters $x_{1}, \ldots, x_{d}$ in $(R, \mathfrak{m})$ is a collection of $d=\operatorname{dim} R$ elements that generate the maximal ideal up 
to radical, i.e., $\mathfrak{m}=\sqrt{\left(x_{1}, \ldots, x_{d}\right)}$. An $R$-algebra $B$ is called big Cohen-Macaulay if every system of parameters

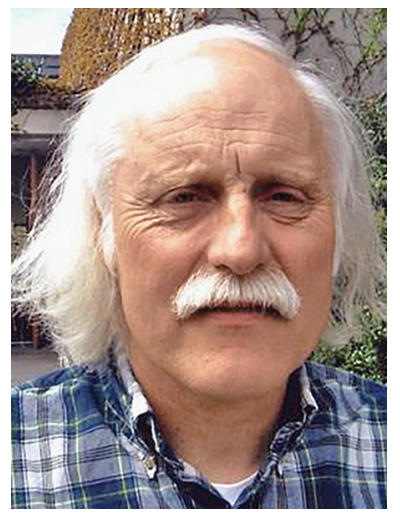

Raymond C. Heitmann. $\underline{x}=x_{1}, \ldots, x_{d}$ of $R$ is a regular sequence on $B$. This means $x_{i+1}$ is a nonzero divisor on $B /\left(x_{1}, \ldots, x_{i}\right)$ and that $m B \neq$ $B$.

Now suppose that $A \longrightarrow$ $R$ is a module-finite extension of Noetherian local rings such that $A$ is regular and $R$ admits a big Cohen-Macaulay algebra. We claim that the map $A \longrightarrow R$ splits. So suppose that $B$ is a big Cohen-Macaulay $R$ algebra; it is easy to see that $B$ is also a big Cohen-Macaulay $A$ algebra (since every system of parameters of $A$ becomes a system of parameters of $R$ ). Because $B$ is big CohenMacaulay and $A$ is regular, $B$ is faithfully flat over $A$ (see [HH92, p.77]). It then follows from the factorization $A \longrightarrow R \longrightarrow B$ that $A \longrightarrow R$ is split (this is similar to the final arguments of the last section).

Therefore the existence of big Cohen-Macaulay algebras implies Theorem 1 for local rings. The general case of Theorem 1 follows from the local case: the evaluation at 1 map $\operatorname{Hom}_{A}(R, A) \longrightarrow A$ is surjective if and only if it is surjective locally. This explains that the existence of big Cohen-Macaulay algebras sits at the top of the diagram at the end of the introduction.

In the case that $R$ contains a field $\mathbb{Q}$ or $\mathbb{Z} / p \mathbb{Z}$, the existence of big Cohen-Macaulay algebras was established by Hochster-Huneke [HH92, HH95]. Hochster had also shown that big Cohen-Macaulay algebras exist in mixed characteristic in dimension three [Hoc02], using ideas of Heitmann's proof of the direct summand conjecture in dimension three [Hei02]. André [And18a] completed this program and showed they exist in mixed characteristic in all dimensions.

We roughly sketch the strategy of the construction of big Cohen-Macaulay algebras following [Hoc94, Hoc02, And18a, HM18]. Suppose that $T$ is an $R$-algebra and that $x_{1}, \ldots, x_{k+1}$ is part of a system of parameters for $R$. Further assume that $t_{1}, \ldots, t_{k+1}$ are elements of $T$ satisfying

( $\quad x_{k+1} t_{k+1}=\sum_{i=1}^{k} x_{i} t_{i}$ but $t_{k+1} \notin\left(x_{1}, \ldots, x_{k}\right) T$.

In other words, $x_{k+1}$ is a zero divisor in $T /\left(x_{1}, \ldots, x_{k}\right) T$ and so $T$ is not (big) Cohen-Macaulay over $R$. We therefore call $(\star)$ a bad relation.

Let $X_{1}, \ldots, X_{k}$ be indeterminates over $T$. We consider

the extension

$$
T \rightarrow T^{\prime}=T\left[X_{1}, \ldots, X_{k}\right] /\left(t_{k+1}-\sum_{i=1}^{k} x_{i} X_{i}\right) .
$$

We have forced $t_{k+1}$ to be inside $\left(x_{1}, \ldots, x_{k}\right) T^{\prime}$, and so the bad relation is trivialized. Such a $T^{\prime}$ is called an algebra modification of $T$. Repeat this process to trivialize all bad relations on $T$ and we obtain a total algebra modification of $T$; call it $T_{1}$. Now we might have new bad relations on $T_{1}$, but we can repeat the whole process above and take a (huge) direct limit. More precisely, we set

$$
B:=\lim _{\longrightarrow}\left(R=T \longrightarrow T_{1} \longrightarrow T_{2} \longrightarrow \cdots\right) .
$$

The above construction guarantees that for every system of parameters $x_{1}, \ldots, x_{d}$ of $R, x_{i+1}$ is a nonzero divisor on $B /\left(x_{1}, \ldots, x_{i-1}\right)$. However, one must show that $\mathfrak{m} B \neq B$. In characteristic $p>0$, this can be proved using the Frobenius map. If $R$ contains $\mathbb{Q}$, a reduction to characteristic $p>0$ technique can be applied (basically by noticing that if $m B=B$ then this must happen at a finite level). Later Hochster [Hoc02] essentially observed that $\mathfrak{m} B \neq B$ as long as we can map $T$ to a certain "almost Cohen-Macaulay algebra"11 (e.g., in characteristic $p>0$, we have $R \longrightarrow R^{1 / p^{\infty}}$ ). Finally in mixed characteristic, André replaced $R^{1 / p^{\infty}}$ by $\left(A_{\infty, \infty} \otimes R\right)^{\prime}$, which is the object that appears in the argument in the proof of Theorem 1 , to prove $\mathfrak{m} B \neq B$.

It turns out that big Cohen-Macaulay algebras have deep connections with singularities. In fact, as we mentioned at the start of the section, if $A$ is regular, then a big Cohen-Macaulay $A$-algebra $B$ is faithfully flat over $A$. From one perspective the role of $B$ is analogous to a resolution of singularities in equal characteristic zero. Suppose that $S$ is (essentially) of finite type over a field $k$ of characteristic zero. Let $\pi: Y \longrightarrow X=\operatorname{Spec} S$ be a resolution of singularities. Grauert-Riemenschneider vanishing [GR70] (a relative version of Kodaira or Kawamata-Viehweg vanishing [Kaw82, Vie82, EV92]) tells us that the higher direct images of the canonical sheaf

$$
R^{i} \pi_{*} \omega_{Y}=0
$$

vanish for $i>0$. By local duality [Har66], this vanishing is equivalent to the following vanishing of local cohomology,

$$
H_{X}^{j}\left(R \pi_{*} \mathcal{O}_{Y}\right)=0
$$

where $j<\operatorname{dim} X, x \in X$ is any closed point, and $\mathbb{W}_{X}^{j}$ denotes sheaf cohomology with support at $X$. In other words, the local cohomology of the complex $R \pi_{*} \mathcal{O}_{Y}$ vanishes except in the top degree. For finitely generated modules, this property of vanishing local cohomology is equivalent to

${ }^{11}$ This means systems of parameters on $R$ are "almost regular sequences"; we omit the detailed definition. 
the Cohen-Macaulay property. In other words, the complex

$$
R \pi_{*} \mathcal{O}_{Y}
$$

is a Cohen-Macaulay algebra, except that it is not an algebra: it lives in the derived category! This was first observed by Roberts [Rob80] (and we omit the non-triviality condition analogous to $m B \neq B$ for simplicity). Many common local applications of Grauert-Riemenschneider vanishing can be proved using the vanishing of local cohomology of $R \pi_{*} \mathcal{O}_{Y}$. Tied up closely with this vanishing are log terminal singularities, which we define in a special case:

Definition 18. A Gorenstein variety $X$ in characteristic zero is called log terminal if the canonical map

$$
H_{X}^{d}\left(\mathcal{O}_{X}\right) \longrightarrow H_{X}^{d}\left(R \pi_{*} \mathcal{O}_{Y}\right)
$$

injects for every $x \in X$ and for some (equivalently every) resolution of singularities $\pi: Y \longrightarrow X$.

Of course, for a big Cohen-Macaulay algebra $B$ over a local ring $S$, we also have the vanishing

$$
H_{\mathfrak{m}}^{i}(B)=0
$$

for the maximal $m \in \operatorname{Spec} S$, and all $i<\operatorname{dim} S$. Furthermore, it follows from work of Smith [Smi94] that in characteristic $p>0$, a Gorenstein local ring $(S, \mathfrak{m})$ is strongly $F$-regular if and only if

$$
H_{\mathfrak{m}}^{d}(S) \longrightarrow H_{\mathfrak{m}}^{d}(B)
$$

is injective for every big Cohen-Macaulay $R$-algebra $B$.

Inspired by this, we introduce the following definition in [MS18b].

Definition 19. Let $(R, \mathfrak{m})$ be a Gorenstein local ring of dimension $d$ and let $B$ be a big Cohen-Macaulay $R$-algebra. Then we say $R$ is big-Cohen-Macaulay-regular with respect to $B$ (or more compactly is $\mathrm{BCM}_{B}$-regular) if the natural map $H_{\mathfrak{m}}^{d}(R) \longrightarrow H_{\mathfrak{m}}^{d}(B)$ is injective.

It turns out that $\mathrm{BCM}_{B}$-regular singularities share many analogous properties of log terminal singularities in equal characteristic 0 or strongly $F$-regular singularities in equal characteristic $p>0$ (again, all based on this vanishing). Furthermore, we apply this to study singularities when the characteristic varies, e.g., families of singularities defined over Spec $\mathbb{Z}$. We refer to [MS18b] for more results in this direction.

\section{An Application to Symbolic Powers}

We discuss another commutative algebraic application of integral perfectoid big Cohen-Macaulay algebras in mixed characteristic [MS18a]. In fact, our proof strategy is directly inspired by the connection between big CohenMacaulay algebras and resolution of singularities and the vanishing theorems discussed above. Let us state the problem.
Suppose $A$ is a Noetherian regular ring (e.g., a polynomial ring over a field or over $\mathbb{Z}$ ). Suppose $Q \subseteq A$ is a prime ideal. For any integer $n>0$ we define the $n t h$ symbolic power of $Q$ to be

$$
Q^{(n)}:=\left(Q^{n} A_{Q}\right) \cap A .
$$

In other words, $Q^{(n)}$ is the set of elements of $A$ (or functions on Spec $A$ ) that vanish to order $n$ at the generic point of $V(Q) \subseteq \operatorname{Spec} A$.

Evidently, $Q^{n} \subseteq Q^{(n)}$ but they are not always equal. A very extensively explored question in commutative algebra studies the difference between $Q^{(n)}$ and $Q^{n}$. For example, when $Q$ is generated by (part of) a regular sequence, a classical result in commutative algebra says that $Q^{n}=Q^{(n)}$ for all $n$. However $Q^{(n)}$ can be much bigger than $Q^{n}$.

Example 20. Let $R=k[x, y, z] /\left(x y-z^{m}\right)$. Then $Q=$ $(x, z)$ is a prime ideal of height one and $x \notin Q^{m}$ (in fact, $x$ is not even in $Q^{2}$ ). However, we see that $x \in Q^{(m)}$ because $x \in Q^{m} R_{Q}$ ( $y$ is a unit in $R_{Q}$ ).

Although the above example shows that $Q^{n}$ and $Q^{(n)}$ can be quite different, a surprising result was obtained by Swanson [Swa00] (see also [HKV09]), who proved essentially that, for any complete local domain $(R, \mathfrak{m})$ and any prime ideal $Q \subseteq R$, there is a constant $k$ (depending on $R$ and $Q$ ) such that $Q^{(k n)} \subseteq Q^{n}$ for all positive integers $n$. In other words, the difference between $Q^{n}$ and $Q^{(n)}$ is bounded "linearly."

For complete regular local rings, we have an even stronger result. The following theorem was proved when our ring contains a field, by Hochster-Huneke [HH02] (see also Ein-Lazarsfeld-Smith [ELS01]), and recently it was extended to mixed characteristic in [MS18a]. Compared with Swanson's result mentioned above, the theorem shows that for regular rings the constant $k$ can be chosen to be the dimension of the ring. In particular it is independent of the ideal $Q$ !

Theorem 21. Let $A$ be a complete regular local ring of dimension $d$. Then for every prime ideal $Q \subseteq A$ and every $n$, we have $Q^{(d n)} \subseteq Q^{n}$.

We briefly explain the strategy of the proof of Theorem 21 in mixed characteristic. The idea is to construct a multiplier ideal like object in mixed characteristic and then use the same strategy as in Ein-Lazarsfeld-Smith [ELSO1]. ${ }^{12}$

For the moment suppose that $A$ is a regular ring of finite type over a field of characteristic 0 (e.g., $A=\mathbb{Q}\left[x_{1}, \ldots, x_{d}\right]$ ). Suppose that $\mathfrak{a} \subseteq A$ is an ideal and $t \in \mathbb{R}_{\geq 0}$ a formal exponent for $\mathfrak{a}$. In this setting we can take a log resolution

${ }^{12}$ A similar object exists in characteristic $p>0$ and is called the test ideal. 
$\pi: Y \longrightarrow$ Spec $A^{13}$ with $\mathfrak{a} \mathcal{O}_{Y}=\mathcal{O}_{Y}(-G)$ and define the multiplier ideal at a maximal ideal $\mathfrak{m} \subseteq A^{14}$ :

$$
\begin{aligned}
\mathcal{J}\left(A_{\mathfrak{m}}, \mathfrak{a}^{t}\right)=\operatorname{Ann}_{A_{\mathfrak{m}}}\left\{\eta \in H_{\mathfrak{m}}^{d}(A) \mid \eta \mapsto 0\right. \\
\left.\quad \in \mathbb{\mathbb { M } _ { \mathfrak { m } } ^ { d }}\left(R \pi_{*} \mathcal{O}_{Y}(\lfloor t G\rfloor)\right)\right\} .
\end{aligned}
$$

This is an ideal of $A_{\mathfrak{m}}$ that measures the singularities of $V(\mathfrak{a}) \subseteq \operatorname{Spec} A$, scaled by $t$, at $\mathfrak{m} \in \operatorname{Spec} A$. Crucially, for the applications to the result on symbolic powers, the multiplier ideal satisfies the following "subadditivity" property [DELO0]:

(†) $\mathcal{J}\left(A_{\mathfrak{m}}, \mathfrak{a}^{t n}\right) \subseteq \mathcal{J}\left(A_{\mathfrak{m}}, \mathfrak{a}^{t}\right)^{n}$ for all positive integers $n$.

This essentially follows from the Kawamata-Viehweg type vanishing result that accompanies the multiplier ideals [Laz04], which can be stated dually as:

$$
\mathbb{W} \mathbb{m}_{\mathfrak{m}}^{i}\left(R \pi_{*} \mathcal{O}_{Y}(\lfloor t G\rfloor)\right)=0 \text { for } i<d .
$$

Philosophy. We examine the definition again. Roughly speaking, the multiplier ideals associated to the pair $\left(A, \mathfrak{a}^{t}\right)$ are elements of $A_{\mathfrak{m}}$ that annihilate all elements in the top local cohomology module $H_{\mathfrak{m}}^{d}(A)$ whose image in

$$
H_{\mathfrak{m}}^{d}\left(R \pi_{*} \mathcal{O}_{Y}\right)
$$

is "almost annihilated" by $\mathfrak{a}^{t}$ (this is made precise in the above definition as $\mathfrak{a}$ in $\mathcal{O}_{Y}$ is just $\mathcal{O}_{Y}(-G)$ ).

Therefore, in order to extend the definition to mixed characteristic and still have the nice properties such as the subadditivity, one needs an object $B$ like $R \pi_{*} \mathcal{O}_{Y}$, which has good vanishing properties and such that one can make sense of, or at least approximate, $\mathfrak{a}^{t}$ in $B$. It turns out that a sufficiently large integral perfectoid big Cohen-Macaulay (or almost big Cohen-Macaulay) algebra will do the job! Below we give a definition of perfectoid multiplier ideal [MS18a] for $A=\mathbb{Z}_{p} \llbracket x_{2}, \ldots, x_{d} \rrbracket$.

Using the fact that ideals are made up of principal ideals, one can essentially reduce the definition to the case where $\mathfrak{a}=(g)$ is principal (we are absolutely hiding subtleties here to keep the definitions cleaner). Let $A_{\infty}$ denote the $p$-adic completion of $A\left[p^{1 / p^{\infty}}, x_{2}^{1 / p^{\infty}}, \ldots, x_{d}^{1 / p^{\infty}}\right]$; this is the same as the definition of $A_{\infty}$ we used in the proof of Theorem 1 in the second section. We then form $A_{\infty, \infty}$ using Theorem 16 for our fixed element $g$ (or in the nonprincipal case, to the generators of $\mathfrak{a}$ ). Then for a fixed real number $t>0$, we can approximate $t$ (from above) by rational numbers of the form $a / p^{e}$, and observe we can identify elements $g^{a / p^{e}} \in A_{\infty, \infty}$ by construction. These $g^{a / p^{e}}$ approximate $g^{t}$.

\footnotetext{
${ }^{13}$ This means $\pi: Y \rightarrow$ Spec $A$ is proper birational, $Y$ is regular, and $\mathfrak{a} \cdot \mathcal{O}_{Y}$ defines a SNC divisor.

${ }^{14}$ This is not the usual definition of multiplier ideals as in [Laz04], but it is equivalent to the usual definition via local and Grothendieck's duality [Har66], see [MS18b, Section 2] for a detailed explanation.
}

In the multiplier ideal definition, we extend $\mathfrak{a}$ to $\mathcal{O}_{Y}$ and then multiply the associated divisor by $t$ (and round as appropriate). We do the same thing here: we define

$$
\begin{aligned}
& \tau\left(A,(g)^{t}\right) \\
& =\operatorname{Ann}_{A}\left\{\eta \in H_{\mathfrak{m}}^{d}(A) \mid p^{1 / p^{\infty}} g^{a / p^{e}} \eta=0 \text { in } H_{\mathfrak{m}}^{d}\left(A_{\infty, \infty}\right)\right\}
\end{aligned}
$$

where $a / p^{e}>t$ approximates $t$ from above.

Although this definition looks a bit technical, it turns out that it satisfies many properties (including the subadditivity property $(\dagger))$ similar to the multiplier ideal $\mathcal{J}\left(A, \mathfrak{a}^{t}\right)$ in characteristic 0 . This allows us to prove Theorem 21. Moreover, the crucial reason that the subadditivity holds for $\boldsymbol{T}\left(A, \mathfrak{a}^{t}\right)$ is because $A_{\infty, \infty}$ is almost big CohenMacaulay. We refer the interested reader to [MS18a, Section 4] for details.

ACKNOWLEDGMENTS. The authors thank the referees as well as Yves André, Bhargav Bhatt, Ray Heitmann, Mel Hochster, and Srikanth Iyengar for numerous helpful comments on this article.

\section{References}

[And18a] André Y. La conjecture du facteur direct, Publ. Math. Inst. Hautes Études Sci. (127):71-93, 2018. MR3814651

[And18b] André Y. Le lemme d'Abhyankar perfectoide, Publ. Math. Inst. Hautes Études Sci. (127):1-70, 2018. MR3814650

[And18c] André Y. Weak functoriality of Cohen-Macaulay algebras, 2018. arXiv: 1801.10010

[AIN18] Avramov LL, Iyengar SB, Neeman A. Big CohenMacaulay modules, morphisms of perfect complexes, and intersection theorems in local algebra, Doc. Math. (23):1601-1619, 2018. MR3890961

[Bha12] Bhatt B. Derived splinters in positive characteristic, Compos. Math., no. 6 (148):1757-1786, 2012. MR2999303

[Bha14a] Bhatt B. Almost direct summands, Nagoya Math. J. (214):195-204, 2014. MR3211823

[Bha14b] Bhatt B. What is ... a perfectoid space?, Notices Amer. Math. Soc., no. 9 (61):1082-1084, 2014. MR3241564

[Bha18] Bhatt B. On the direct summand conjecture and its derived variant, Invent. Math., no. 2 (212):297-317, 2018. MR3787829

[BMS18] Bhatt B, Morrow M, Scholze P. Integral $p$-adic Hodge theory, Publ. Math. Inst. Hautes Études Sci. (128):219-397, 2018, DOI 10.1007/s10240-019-00102-z. MR3905467

[DEL00] Demailly J-P, Ein L, Lazarsfeld R. A subadditivity property of multiplier ideals, Michigan Math. J. (48):137156, 2000. Dedicated to William Fulton on the occasion of his 60th birthday. MR1786484 (2002a:14016)

[Dut87] Dutta SP. On the canonical element conjecture, Trans. Amer. Math. Soc., no. 2 (299):803-811, 1987. MR869233 
[ELS01] Ein L, Lazarsfeld R, Smith KE. Uniform bounds and symbolic powers on smooth varieties, Invent. Math., no. 2 (144):241-252, 2001. MR1826369 (2002b:13001)

[EV92] Esnault H, Viehweg E. Lectures on vanishing theorems, DMV Seminar, vol. 20, Birkhäuser Verlag, Basel, 1992. MR1193913 (94a:14017)

[EG81] Evans E, Griffith P. The syzygy problem, Ann. of Math. (2), no. 2 (114):323-333, 1981. MR632842

[Fal88] Faltings G. $p$-adic Hodge theory, J. Amer. Math. Soc., no. 1 (1):255-299, 1988. MR924705

[Fal02] Faltings G. Almost étale extensions, Astérisque (279):185-270, 2002. Cohomologies $p$-adiques et applications arithmétiques, II. MR1922831

[Gab18] Gabber O. Observations made after the MSRI workshop on homological conjectures, 2018. https://docs.google.com/viewer?ur 1=

https://www.msri.org/workshops/842

/scheduTes/23854/documents/3322/assets /31362.

[GR03] Gabber O, Ramero L. Almost ring theory, Lecture Notes in Mathematics, vol. 1800, Springer-Verlag, Berlin, 2003.

[GR04] Gabber O, Ramero L. Foundations for almost ring theory, 2004. arXiv:0409584.

[GR70] Grauert H, Riemenschneider O. Verschwindungssätze für analytische Kohomologiegruppen auf komplexen Räumen, Invent. Math. (11):263-292, 1970. MR0302938 (46 \#2081)

[Har98] Hara N. A characterization of rational singularities in terms of injectivity of Frobenius maps, Amer. J. Math., no. 5 (120):981-996, 1998. MR1646049 (99h:13005)

[HW02] Hara N, Watanabe K-I. F-regular and F-pure rings vs. log terminal and log canonical singularities, J. Algebraic Geom., no. 2 (11):363-392, 2002. MR1874118 (2002k:13009)

[Har66] Hartshorne R. Residues and duality, Lecture notes of a seminar on the work of A. Grothendieck, given at Harvard 1963/64. With an appendix by P. Deligne. Lecture Notes in Mathematics, No. 20, Springer-Verlag, Berlin, 1966. MR0222093 (36 \#5145)

[Har79] Hartshorne R. Algebraic vector bundles on projective spaces: a problem list, Topology, no. 2 (18):117-128, 1979. MR544153

[HM18] Heitmann R, Ma L. Big Cohen-Macaulay algebras and the vanishing conjecture for maps of Tor in mixed characteristic, Algebra Number Theory, no. 7 (12):1659-1674, 2018. MR3871506

[Hei02] Heitmann RC. The direct summand conjecture in dimension three, Ann. of Math. (2), no. 2 (156):695-712, 2002. MR1933722 (2003m:13008)

[Hoc73] Hochster M. Contracted ideals from integral extensions of regular rings, Nagoya Math. J. (51):25-43, 1973. MR0349656 (50 \#2149)

[Hoc75] Hochster M. Topics in the homological theory of modules over commutative rings, Published for the Conference Board of the Mathematical Sciences by the American Mathematical Society, Providence, R.I., 1975. Expository lectures from the CBMS Regional Conference held at the University of Nebraska, Lincoln, Neb., June 24-28, 1974, Conference Board of the Mathematical Sciences Regional Con- ference Series in Mathematics, No. 24. MR0371879 (51 \#8096)

[Hoc83] Hochster M. Canonical elements in local cohomology modules and the direct summand conjecture, J. Algebra, no. 2 (84):503-553, 1983. MR723406

[Hoc94] Hochster M. Solid closure. Commutative algebra: syzygies, multiplicities, and birational algebra (South Hadley, MA, 1992): Amer. Math. Soc., Providence, RI; 1994:103-172. MR1266182

[Hoc97] Hochster M. Nonnegativity of intersection multiplicities in ramified regular local rings following Gabber/De Jong/Berthelot, 1997. available at: http://www. math. 1sa.umich. edu/ hochster $/ \mathrm{mu} 7 \mathrm{t} . \mathrm{ps}$, unpublished notes.

[Hoc02] Hochster M. Big Cohen-Macaulay algebras in dimension three via Heitmann's theorem, J. Algebra, no. 2 (254):395-408, 2002.

[Hoc04] Hochster M. Current state of the homological conjectures: Five talks of a VIGRE-funded minicourse at the University of Utah, 2004. available at: http://www.math.utah.edu/vigre /minicourses/algebra/hochster.pdf.

[Hoc17] Hochster M. Homological conjectures and lim Cohen-Macaulay sequences. Homological and computational methods in commutative algebra: Springer, Cham; 2017:173-197. MR3751886

[HH90] Hochster M, Huneke C. Tight closure, invariant theory, and the Briançon-Skoda theorem, J. Amer. Math. Soc., no. 1 (3):31-116, 1990. MR1017784 (91g:13010)

[HH92] Hochster M, Huneke C. Infinite integral extensions and big Cohen-Macaulay algebras, Ann. of Math. (2), no. 1 (135):53-89, 1992. MR1147957 (92m:13023)

[HH94] Hochster M, Huneke C. F-regularity, test elements, and smooth base change, Trans. Amer. Math. Soc., no. 1 (346):1-62, 1994. MR1273534(95d:13007)

[HH95] Hochster M, Huneke C. Applications of the existence of big Cohen-Macaulay algebras, $A d v$. Math., no. 1 (113):45-117, 1995. MR1332808

[HH02] Hochster M, Huneke C. Comparison of symbolic and ordinary powers of ideals, Invent. Math., no. 2 (147):349-369, 2002. MR1881923 (2002m:13002)

[HKV09] Huneke C, Katz D, Validashti J. Uniform equivalence of symbolic and adic topologies, Illinois J. Math., no. 1 (53):325-338, 2009. MR2584949

[Kaw82] Kawamata Y. A generalization of KodairaRamanujam's vanishing theorem, Math. Ann., no. 1 (261):43-46, 1982. MR675204 (84i:14022)

[KL15] Kedlaya KS, Liu R. Relative $p$-adic Hodge theory: foundations, Astérisque (371):239, 2015. MR3379653

[Kov00] Kovács SJ. A characterization of rational singularities, Duke Math. J., no. 2 (102):187-191, 2000. MR1749436 (2002b:14005)

[Laz04] Lazarsfeld R. Positivity in algebraic geometry. II, Ergebnisse der Mathematik und ihrer Grenzgebiete. 3. Folge. A Series of Modern Surveys in Mathematics [Results in Mathematics and Related Areas. 3rd Series. A Series of Modern Surveys in Mathematics], vol. 49, Springer-Verlag, Berlin, 2004. Positivity for vector bundles, and multiplier ideals. MR2095472 (2005k:14001b) 
[MS18a] Ma L, Schwede K. Perfectoid multiplier/test ideals in regular rings and bounds on symbolic powers, Invent. Math., no. 2 (214):913-955, 2018. MR3867632

[MS18b] Ma L, Schwede K. Singularities in mixed characteristic via big Cohen-Macaulay algebras, 2018. arXiv:1806.09567.

[MS97] Mehta V, Srinivas V. A characterization of rational singularities, Asian J. Math., no. 2 (1):249-271, 1997. MR1491985 (99e:13009)

[PS73] Peskine C, Szpiro L. Dimension projective finie et cohomologie locale. Applications à la démonstration de conjectures de M. Auslander, H. Bass et A. Grothendieck, Inst. Hautes Études Sci. Publ. Math. (42):47-119, 1973. MR0374130 (51 \#10330)

[Rob80] Roberts P. Cohen-Macaulay complexes and an analytic proof of the new intersection conjecture, J. Algebra, no. 1 (66):220-225, 1980. MR591254

[Rob87] Roberts P. Le théorème d'intersection, C. R. Acad. Sci. Paris Sér. I Math., no. 7 (304):177-180, 1987. MR880574

[Rob89] Roberts P. Intersection theorems. Commutative algebra (Berkeley, CA, 1987): Springer, New York; 1989:417436. MR1015532

[Sch12] Scholze P. Perfectoid spaces, Publ. Math. Inst. Hautes Études Sci. (116):245-313, 2012. http://dx.doi.org/ 10.1007/s10240-012-0042-x. MR3090258

[Shi17] Shimomoto K. Integral perfectoid big CohenMacaulay algebras via André's theorem, 2017. arXiv: 1706.06946 , to appear in Math. Ann.

[Sil09] Silverman JH. The arithmetic of elliptic curves, Second, Graduate Texts in Mathematics, vol. 106, Springer, Dordrecht, 2009. MR2514094 (2010i:11005)

[Sin99] Singh AK. Q-Gorenstein splinter rings of characteristic $p$ are F-regular, Math. Proc. Cambridge Philos. Soc., no. 2 (127):201-205, 1999. MR1735920 (2000j:13006)

[Smi94] Smith K. Tight closure of parameter ideals, Invent. Math., no. 1 (115):41-60, 1994. MR1248078 (94k:13006)

[Smi97] Smith KE. $F$-rational rings have rational singularities, Amer. J. Math., no. 1 (119):159-180, 1997. MR1428062 (97k:13004)

[Swa00] Swanson I. Linear equivalence of ideal topologies, Math. Z., no. 4 (234):755-775, 2000. MR1778408

[Vie82] Viehweg E. Vanishing theorems, J. Reine Angew. Math. (335):1-8, 1982. MR667459 (83m:14011)

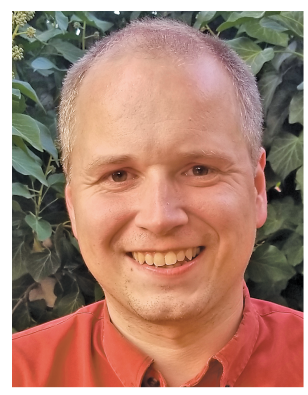

Karl Schwede

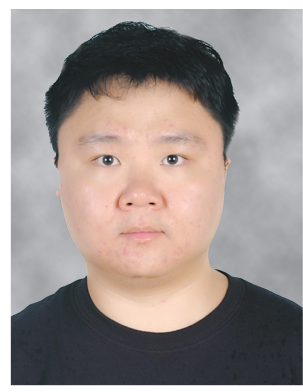

Linquan $\mathrm{Ma}$

\section{Credits}

Photo of Melvin Hochster is courtesy of Melvin Hochster.

Photo of Yves André is courtesy of Yves André.

Photo of Ofer Gabber is courtesy of Renate Schmid, Copyright

is with MFO [CC BY-SA 2.0 de (https://

creativecommons.org/1icenses/by-sa/2.0

/de/deed.en)].

Photo of Paul C. Roberts is courtesy of Paul C. Roberts.

Photo of Karen E. Smith is ${ }^{\odot}$ Eric Bronson, Michigan Photography.

Photo of Kei-ichi Watanabe is courtesy of Ernst Kunz.

Photo of Bhargav Bhatt is courtesy of Bhargav Bhatt.

Photo of Craig Huneke is courtesy of MFO [CC BY-SA 2.0 de

(https://creativecommons .org/7icenses/by-

sa/2.0/de/deed.en)].

Photo of Raymond C. Heitmann is $\odot$ Jürgen Herzog.

Photo of Linquan Ma is courtesy of Linquan Ma.

Photo of Karl Schwede is courtesy of Karl Schwede.

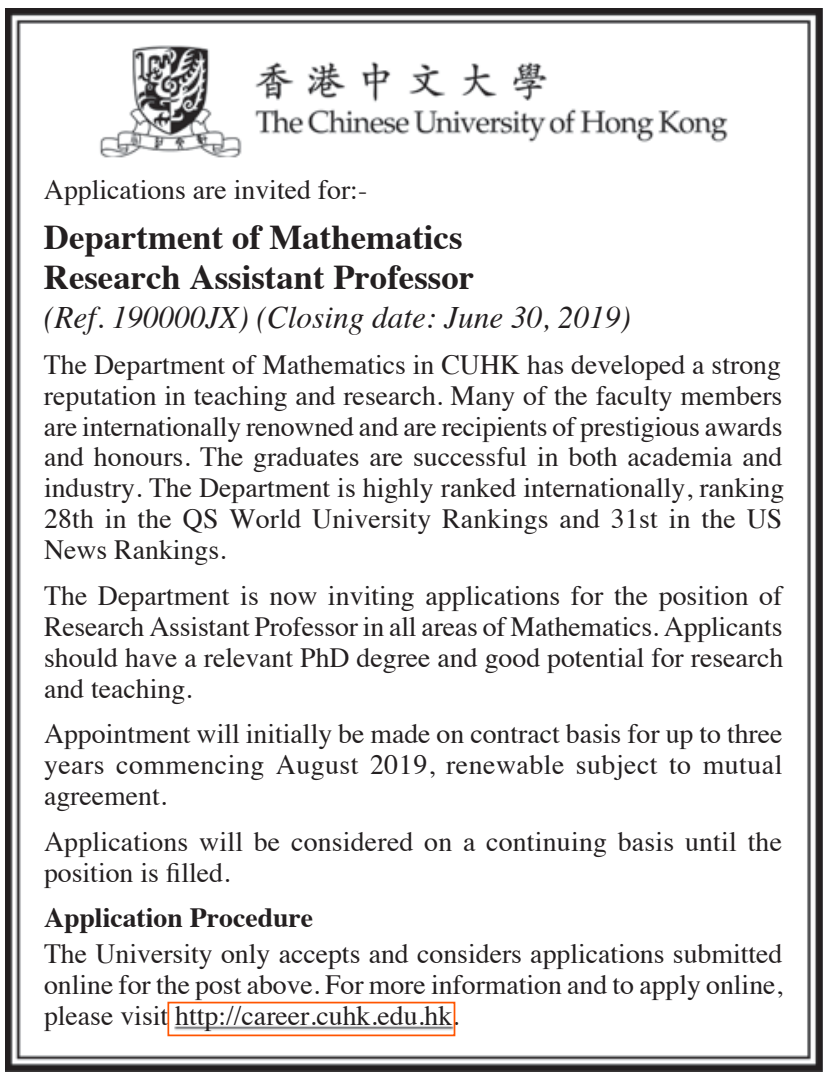

